\title{
New prognostic biomarker CMTM3 in low grade glioma and its immune infiltration
}

\author{
Shoubin $\mathrm{Li}^{1}$, Peng Gao ${ }^{1}$, Xingliang Dai ${ }^{1}$, Lei Ye ${ }^{1}$, Zhongyong Wang ${ }^{2}$, Hongwei Cheng ${ }^{1}$ \\ ${ }^{1}$ Department of Neurosurgery, the First Affiliated Hospital of Anhui Medical University, Hefei, China; ${ }^{2}$ Department of Neurosurgery, the Second \\ Affiliated Hospital of Soochow University, Suzhou, China \\ Contributions: (I) Conception and design: H Cheng, Z Wang; (II) Administrative support: H Cheng, Z Wang; (III) Provision of study materials or \\ patients: S Li, P Gao, X Dai; (IV) Collection and assembly of data: S Li, L Ye; (V) Data analysis and interpretation: S Li, L Ye; (VI) Manuscript \\ writing: All authors; (VII) Final approval of manuscript: All authors. \\ Correspondence to: Zhongyong Wang. Department of Neurosurgery, the Second Affiliated Hospital of Soochow University, 1055 Sanxaing Road, \\ Suzhou 215004, China. Email: wangzhongyong@suda.edu.cn; Hongwei Cheng. Department of Neurosurgery, the First Affiliated Hospital of Anhui \\ Medical University, 218 Jixi Road, Hefei 230022, China. Email: hongwei.cheng@ahmu.edu.cn.
}

Background: The CKLF-like MARVEL transmembrane domain-containing 3 (CMTM3) is differentially expressed in a variety of tumors and closely related to tumor occurrence and progression. The expression of CMTM3 was significantly elevated in glioma compared with normal brain tissue, to explore the potential function of CMTM3 in the prognosis and immune infiltration of glioma has certain clinical significance.

Methods: The tumor data in this study were derived from the sequencing data of various tumors in The Cancer Genome Atlas (TCGA) database. Low-grade glioma (LGG) data in the TCGA database include sequencing and clinical data. Clinical data mainly include survival time, survival outcome, age, WHO classification and other information. Sequencing data for normal tissues were obtained from the Genotype Tissue Expression (GTEx) database. Statistical analyses were mainly performed using bioinformatics tools and the corresponding $\mathrm{R}$ software (version 3.6.3). The Mann-Whitney $\mathrm{U}$ test (Wilcoxon rank sum test) was used to compare the expression differences between the tumor group and the normal group. Survival analysis was conducted using log-rank test to compare whether the overall survival (OS) time was statistically different between the CMTM3 high and low expression groups. The Tumor Immunity Estimation Resource (TIMER) database was used for immune infiltration analysis.

Results: The results showed that the expression of CMTM3 in World Health Organization (WHO) II and WHO III gliomas was significantly higher than that of normal tissues $(\mathrm{P}<0.05)$. Glioma with high CMTM3 expression showed a lower overall survival (OS) $(\mathrm{P}<0.05)$. Gene enrichment analysis showed that CMTM3 was significantly enriched in 4 pathways (FDR $<0.25, \mathrm{P}<0.05$ ). A high correlation was detected between CMTM3 and a variety of immune cells. CMTM3 is highly correlated with macrophages $(\mathrm{r}=0.536$, $\mathrm{P}=1.31 \mathrm{e}-36)$, dendritic cells ( $\mathrm{r}=0.546, \mathrm{P}=2.85 \mathrm{e}-38)$, CD4+ T cells ( $\mathrm{r}=0.517, \mathrm{P}=6.17 \mathrm{e}-34)$.

Conclusions: The CMTM3 gene can be used as a potential prognostic marker for WHO grade II and WHO grade III glioma, is related to the immune infiltration in glioma microenvironment, and may became a new immunotherapy target.

Keywords: CKLF-like MARVEL transmembrane domain-containing 3 (CMTM3); bioinformatics; The Cancer Genome Atlas (TCGA); glioma

Submitted Dec 15, 2021. Accepted for publication Feb 18, 2022.

doi: $10.21037 /$ atm-22-526

View this article at: https://dx.doi.org/10.21037/atm-22-526 


\section{Introduction}

The revision of the World Health Organization (WHO) classification of central nervous system tumors has led to major changes in the routine diagnosis and treatment of patients with glioma (1). However, glioma remains a malignant tumor of the central nervous system with high recurrence rate, high fatality rate, and poor prognosis (2). The prognosis of different types and grades of glioma varies greatly. Although a variety of comprehensive treatment methods are used in combination, such as surgery, radiotherapy, chemotherapy, immunotherapy, electric field therapy, and neutron therapy, the treatment effect of glioma is still unsatisfactory. Therefore, it is necessary to find new treatments. CKLF-like MARVEL transmembrane domaincontaining 3 (CMTM3) has a high correlation with immune cells, and it can be used as a potential immunotherapy target. There are many studies on prognostic markers of glioma, but there are few biomarkers recognized and widely used in the clinic. Most prognostic markers can only be used as prognostic markers alone, and their correlation with immune cells is also low, making it difficult to serve as potential therapeutic targets. As a new prognostic marker, CMTM3 can not only judge the prognosis, but also is expected to become a potential immune target. It has a high clinical application prospect.

The expression and function of CMTM3 in different tumor types are quite different. It shows tumor suppressor effect in some tumors while showing oncogene characteristics in other tumors. Some studies have suggested that CMTM3 inhibits tumor cell invasion and migration in gastric cancer, liver cancer, prostate cancer, and testicular cancer, and plays the role of tumor suppressor genes (3-6). However, some others studies have shown that CMTM3 overexpression predicts poor survival and promotes proliferation and migration of pancreatic cancer (7). Yang et al. (8) predicted the potential of CMTM3 as a candidate marker for glioblastoma. Delic et al. (9) predicted the potential of CMTM3 to promote tumor cell invasion by analyzing the correlation between the CMTM family and the pathogenesis of glioblastoma. However, the correlation between CMTM3 and low-grade glioma (WHO grade II and III) and its immune infiltration are still unclear.

In this study, we used The Cancer Genome Atlas (TCGA) data to analyze the expression difference of CMTM3 in WHO grade II and WHO grade III glioma compared to normal tissues. We analyzed the relationship between CMTM3 and clinicopathological factors. Gene set enrichment analysis (GSEA) was used to explore related biological processes and pathways regulated by CMTM3. The Tumor Immune Estimation Resource (TIMER) database was used to analyze the correlation between CMTM3 and immune infiltration. The primary aim of this study was to demonstrate the prognostic role of $C M T M 3$ as a new prognostic biomarker for improving survival predictions for WHO grade II-III glioma patients. We present the following article in accordance with the REMARK reporting checklist (available at https://atm. amegroups.com/article/view/10.21037/atm-22-526/rc).

\section{Methods}

\section{Data acquisition}

The RNA-seq data for glioma was obtained from TCGA database (https://portal.gdc.cancer.gov/). The downloaded data was all WHO II and WHO III glioma data, without glioblastoma data. The RNA-seq data for normal samples was from the Genotype Tissue Expression (GTEx) database. The data of patients with significant missing information were excluded. We used two sets of data to analyze the difference in the expression of CMTM3 in glioma and normal tissues. This study complied with the publication guidelines set by TCGA. Ethical approval and informed consent of patients were not required. The study was conducted in accordance with the Declaration of Helsinki (as revised in 2013).

\section{Analysis of TIMER database}

The TIMER (http://cistrome.dfci.harvard.edu/TIMER/) is a comprehensive database (10) which can be used to systematically analyze the immune infiltration of different types of cancer. The current version of TIMER contains 10,009 samples from 23 cancer types from the TCGA data set to estimate the abundance of immune infiltration. We used the TIMER online database to analyze the association of $C M T M 3$ with immune infiltration.

\section{Analysis of gene set enrichment}

GSEA is a computational method used to determine whether a set of a priori defined genes show statistically significant differences between 2 biological states (11). The GSEA revealed a significantly enriched gene set [false discovery rate $(\mathrm{FDR})<0.25, \mathrm{P}$ value $<0.05]$ in $C M T M 3$. 
GSEA analysis looked for signaling pathways that CMTM3 may be involved in affecting the progression of LGG (WHO grade II and WHO grade III).

\section{Gene Expression Profiling Interaction Analysis 2 (GEPIA2) database}

The GEPIA2 database was developed by Peking University as a new version of the original GEPIA. It is used to analyze the RNA sequencing expression data of 9,736 tumors and 8,587 normal samples from TCGA and GTEx projects (12). It can analyze the TCGA database online. We used it to further verify that the expression difference of CMTM3 in LGG and normal tissues was statistically significant.

\section{Statistical analysis}

Statistical analysis and visualization were performed using the corresponding $\mathrm{R}$ package ( $\mathrm{R}$ version 3.6.3; https:// www.r-project.org/). CMTM3 in LGG (WHO grade II and WHO grade III) and GTEX did not conform to normal distribution $(\mathrm{P}<0.05$ for normality test), so Mann-Whitney $\mathrm{U}$ test was used. The Mann-Whitney $\mathrm{U}$ test (Wilcoxon rank sum test) was used to compare the expression differences between the tumor group and the normal group. Survival analysis was conducted using log-rank test to compare whether the overall survival (OS) time was statistically different between the CMTM3 high and low expression groups. The survminer package was used to draw survival curves. GO and KEGG enrichment analysis of CMTM3related genes. Two different immune infiltration algorithms were used in the analysis of immune infiltration, namely the single-sample (ss) GSEA immune infiltration algorithm and the immune algorithm built in the TIMER database. The two different algorithms illustrate the correlation between CMTM 3 and immune infiltration. $\mathrm{P}<0.05$ was considered statistically significant. Significance levels: ${ }^{*} \mathrm{P}<0.05$, ${ }^{* *} \mathrm{P}<0.01,{ }^{* * *} \mathrm{P}<0.001$.

\section{Results}

\section{CMTM3 is bighly expressed in WHO grade II and III gliomata}

We downloaded the RNA-seq data of all tumor samples in TCGA database and the RNA-seq data of normal tissue samples in the GTEx database, and compared the transcription level of CMTM3 in various tumors. It was found that $C M T M 3$ was highly expressed in most tumors (Figure 1A). The ggplot2 package was used to visualize the data, and the expression of CMTM3 in glioma and normal tissues was significantly different (Figure 1B). The GEPIA2 and Gene Expression Omnibus (GEO) databases showed that CMTM3 is highly expressed in low-grade gliomas (LGG) (Figure 1C).

\section{Relationship between CMTM3 expression and clinical features of glioma}

The clinical information of WHO grade II and III glioma was downloaded from TCGA database (Table 1). We compared whether there are significant differences in the expression of $C M T M 3$ in different clinical features between WHO grade II and III glioma. The results showed that the expression of CMTM3 in grade II and III glioma was significantly different, and as the grade of glioma increased, the expression of CMTM3 also increased (Figure 2A). The isocitrate dehydrogenase (IDH) in WHO grade II and III glioma was divided into wild type (WT) and mutant type (MT). The difference in CMTM3 expression between the two groups was statistically significant (Figure 2B). The expression of CMTM3 in the $1 \mathrm{p} / 19 \mathrm{q}$ co-deletion group was overall lower than that of the no co-deletion group (Figure 2C). The expression level of CMTM3 in the group older than 40 years was higher than that in the group younger than 40 years (Figure 2D).

\section{CMTM3 expression on the prognosis of WHO grade II and III gliomas}

A time-dependent receiver operating characteristic (ROC) curve showed that the area under the curve (AUC) of CMTM3 predicting the 1- and 3-year survival outcomes of WHO grade II and III glioma patients were 0.821 and 0.750 , indicating that $C M T M 3$ can predict patient survival well (Figure 3A). Kaplan-Meier survival analysis showed that $C M T M 3$ expression was correlated with OS in $\mathrm{WHO}$ grade II and III glioma patients (Figure 3B). High CMTM3 expression indicated a poor prognosis and a lower survival rate. The survival of CMTM3 in different clinical subgroups also had significant differences. Patients with WHO grade III gliomas with high CMTM3 expression had lower OS and less favorable prognosis. However, WHO grade III gliomas with low CMTM3 expression had a more favorable prognosis (Figure 3C). Additionally, CMTM3 was highly expressed in IDH wild-type gliomas, and the OS rate was 


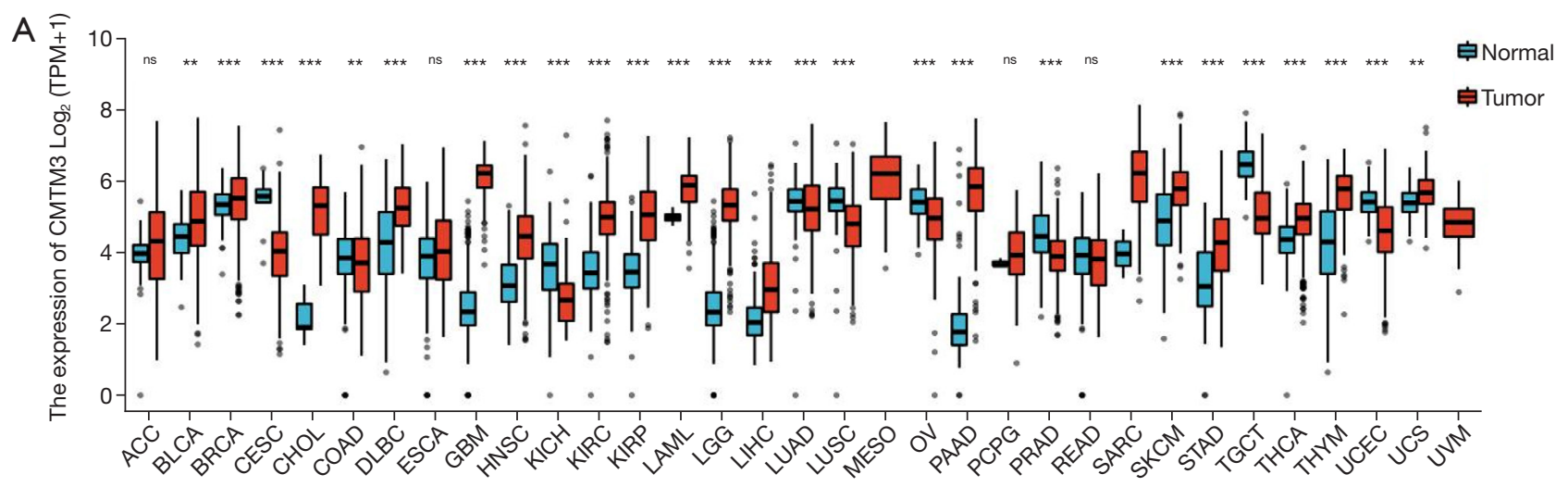

\section{B}

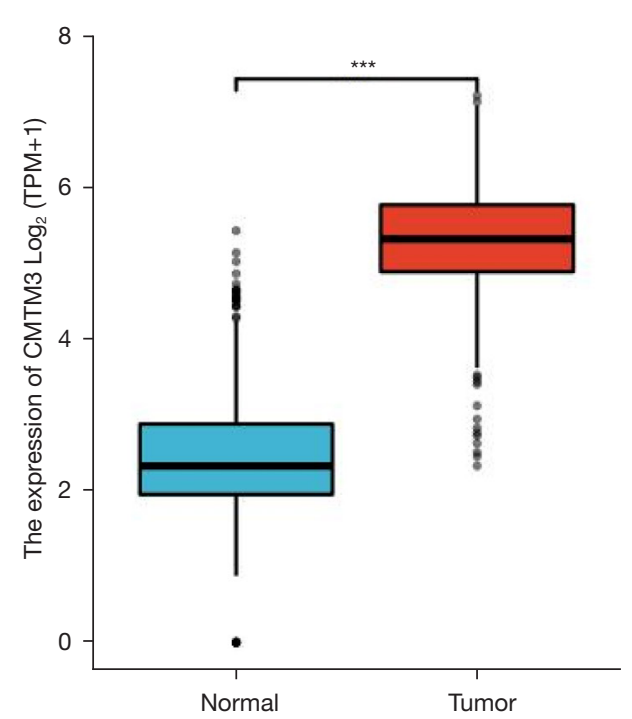

C

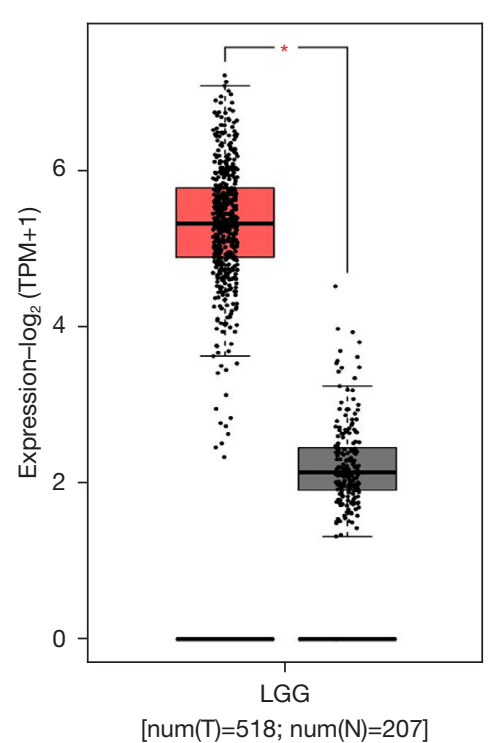

Figure 1 CMTM3 mRNA expression levels. (A) CMTM3 mRNA expression levels in different tumor types from TCGA database; ns, $\mathrm{P} \geq 0.05$; (B) CMTM3 expression levels in glioma and matched normal tissues; (C) GEPIA database shows differences in the expression of CMTM3 in low grade glioma and normal tissues. * $\mathrm{P}<0.05$; ${ }^{* *}, \mathrm{P}<0.01$; **, $\mathrm{P}<0.001$; ns, $\mathrm{P}>0.05$. ACC, adrenocortical carcinoma; $\mathrm{BLCA}$, bladder urothelial carcinoma; BRCA, breast invasive carcinoma; CESC, cervical squamous cell carcinoma and endocervical adenocarcinoma; CHOL, cholangiocarcinoma; COAD, colon adenocarcinoma; DLBC, lymphoid neoplasm diffuse large B-cell lymphoma; ESCA, esophageal carcinoma; GBM, glioblastoma multiforme; HNSC, head and neck squamous cell carcinoma; KICH, kidney chromophobe; KIRC, kidney renal clear cell carcinoma; KIRP, kidney renal papillary cell carcinoma; LAML, acute myeloid leukemia; LGG, low grade glioma; LIHC, liver hepatocellular carcinoma; LUAD, lung adenocarcinoma; LUSC, lung squamous cell carcinoma; MESO, mesothelioma; OV, ovarian serous cystadenocarcinoma; PAAD, pancreatic adenocarcinoma; PCPG, pheochromocytoma and paraganglioma; PRAD, prostate adenocarcinoma; READ, rectum adenocarcinoma; SARC, sarcoma; SKCM, skin cutaneous melanoma; STAD, stomach adenocarcinoma; TGCT, testicular germ cell tumors; THCA, thyroid carcinoma; THYM, thymoma; UCEC, uterine corpus endometrial carcinoma; UCS, uterine carcinosarcoma; UVM, uveal melanoma; CMTM3, CKLF-like MARVEL transmembrane domain-containing 3; mRNA, messenger RNA; TCGA, The Cancer Genome Atlas; GEPIA, gene expression profiling analysis.

lower (Figure 3D). The OS rate of CMTM3 high expression in the $1 \mathrm{p} / 19 \mathrm{q}$ non-codeletion glioma subgroup was lower than that of the low expression group (Figure 3E). In the subgroup of WHO grade II and III glioma patients older than 40 years, the OS curve showed that the prognosis difference between the two groups with high and low CMTM3 expression 
Table 1 Clinical related data of glioma

\begin{tabular}{lcc}
\hline Characteristic & Low expression of CMTM3 (N=264) & High expression of CMTM3 (N=264) \\
\hline WHO grade, $n(\%)$ & $135(28.9)$ & $89(19.1)$ \\
G2 & $95(20.3)$ & $148(31.7)$ \\
G3 & & $<0.001$ \\
IDH status, $n(\%)$ & $17(3.2)$ & $80(15.2)$ \\
WT & $244(46.5)$ & $184(35.0)$ \\
Mut & & $72(13.6)$ \\
1p/19q codeletion, $n(\%)$ & $99(18.8)$ & $192(36.4)$ \\
Codel & $165(31.2)$ & 0.016 \\
Non-codel & & 1601 \\
\hline
\end{tabular}

IDH, isocitrate dehydrogenase; CMTM3, CKLF-like MARVEL transmembrane domain-containing 3; WT, wild-type; Mut, mutant; WHO, World Health Organization; codel, codeletion.

A

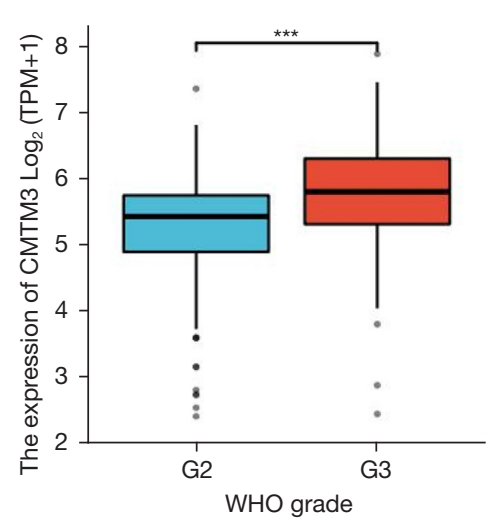

C

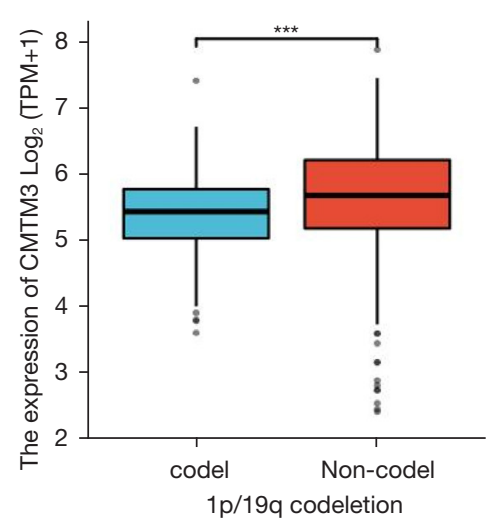

B

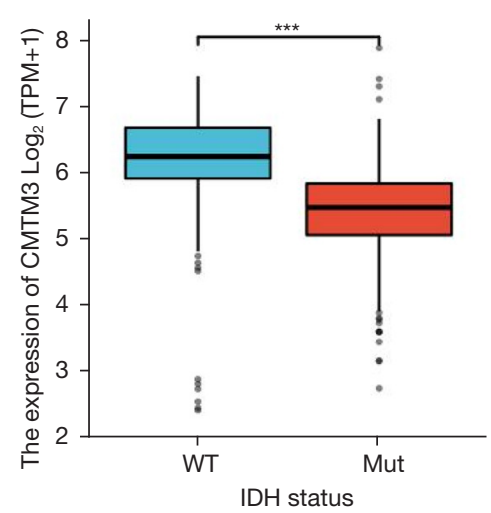

$\mathrm{D}$

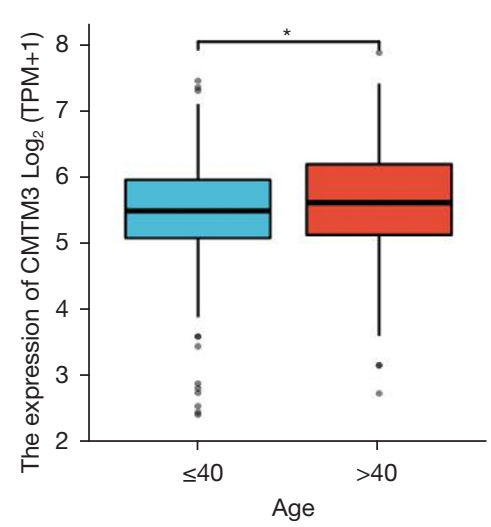

Figure 2 CMTM3 expression and clinical features in glioma. (A) WHO grade; (B) IDH status; (C) 1p/19qcondeletion; (D) age. *, P<0.05; ***, $\mathrm{P}<0.001$. CMTM3, CKLF-like MARVEL transmembrane domain-containing 3; WHO, World Health Organization. 
A

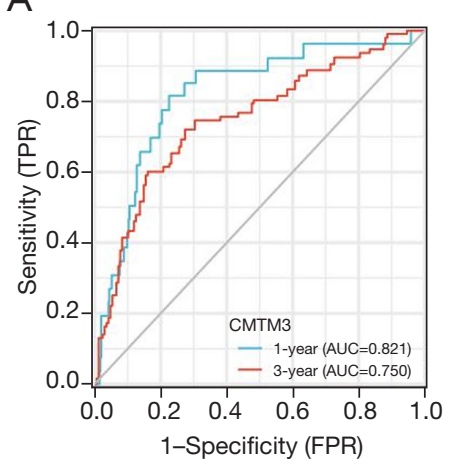

D

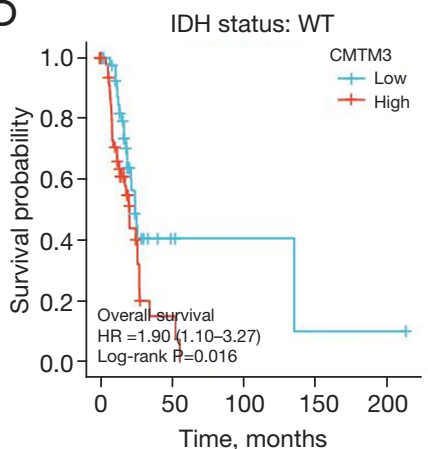

B

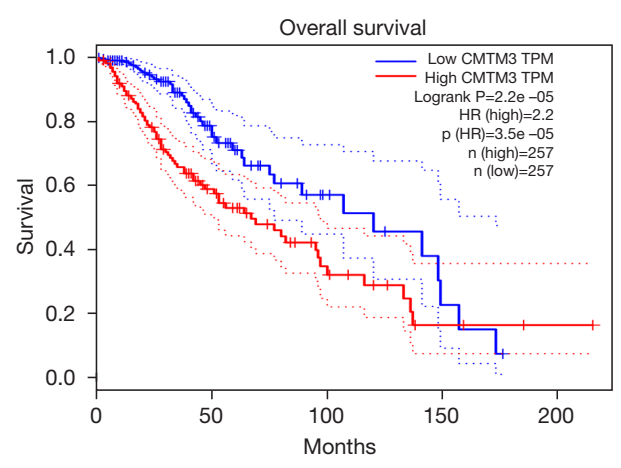

E

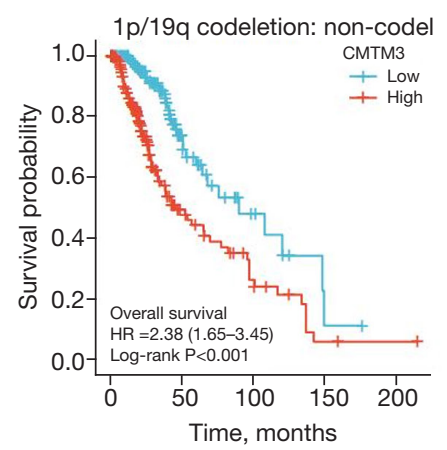

C

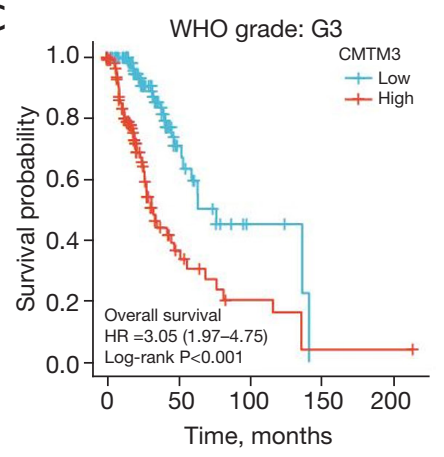

$\mathrm{F}$

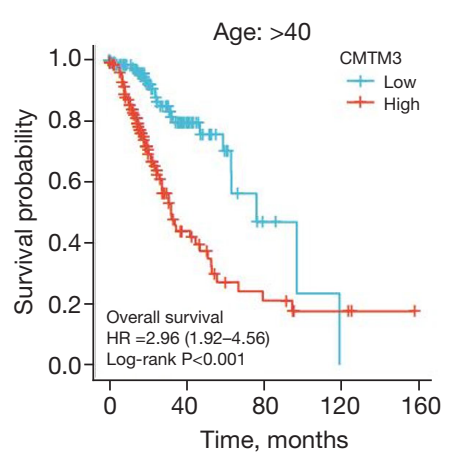

Figure 3 Prognostic analysis of CMTM3. (A) Time ROC curves; (B) overall survival curve of differential CMTM3 expression in WHO grade II and III glioma; (C) OS curve of WHO grade III glioma subgroup; (D) OS curve of IDH wild-type glioma; (E) OS curve of 1p/19q non-codeletion glioma subgroup; (F) OS curve of glioma subgroup over 40 years old. CMTM3, CKLF-like MARVEL transmembrane domain-containing 3; OS, overall survival; IDH, isocitrate dehydrogenase.

was also statistically significant (Figure 3F).

\section{GSEA enrichment analysis of CMTM3 enriched patbway in $L G G$}

We conducted GSEA to study the biological function of CMTM3 in WHO grade II and III glioma. The GSEA showed 4 pathways where CMTM3 was significantly enriched (FDR $<0.25, \mathrm{P}$ value $<0.05)$ (Figure 4$)$. The pathways included the microglial pathogen phagocytosis pathway, PLK1 pathway, IL2RB pathway, and P53 signal pathway.

\section{Differential gene expression and gene association analysis of CMTM3 in LGG}

Comparative analysis of differential gene expression in CMTM3 high expression and low expression groups of LGG showed that there were 138 genes with a difference factor (log2FoldChange) greater than 2 and 128 genes with a difference of less than 2 (Figure 5A, Table 2). The Gene Ontology (GO) analysis and Kyoto Encyclopedia of Genes and Genomes (KEGG) analysis of the differential genes are shown Table 3. Genetic correlation analysis showed that the correlations between CMTM3 and CMTM7, BLNK, LCP2, CKLF, CMTM6, CMTM1, CMTM2, CMTM4, TTC7B, EEA1, SF3B3, KYNU, ZNF232, TM4SF20, and GPR68 had significant differences (Figure $5 B$ ). The CMTM3 and the above-mentioned gene protein-protein interaction (PPI) network are shown in Figure 5 C.

\section{Immune infiltration analysis of CMTM3 in LGG}

The ssGSEA immune infiltration algorithm was used to calculate the correlation between CMTM3 and 24 immune cells (Figure 6A). The results showed that CMTM3 had the highest correlation with activated dendritic cells $(\mathrm{aDC}$; $\mathrm{r}=0.472, \mathrm{P}<0.001)$ and macrophages $(\mathrm{r}=0.456, \mathrm{P}<0.001)$. 

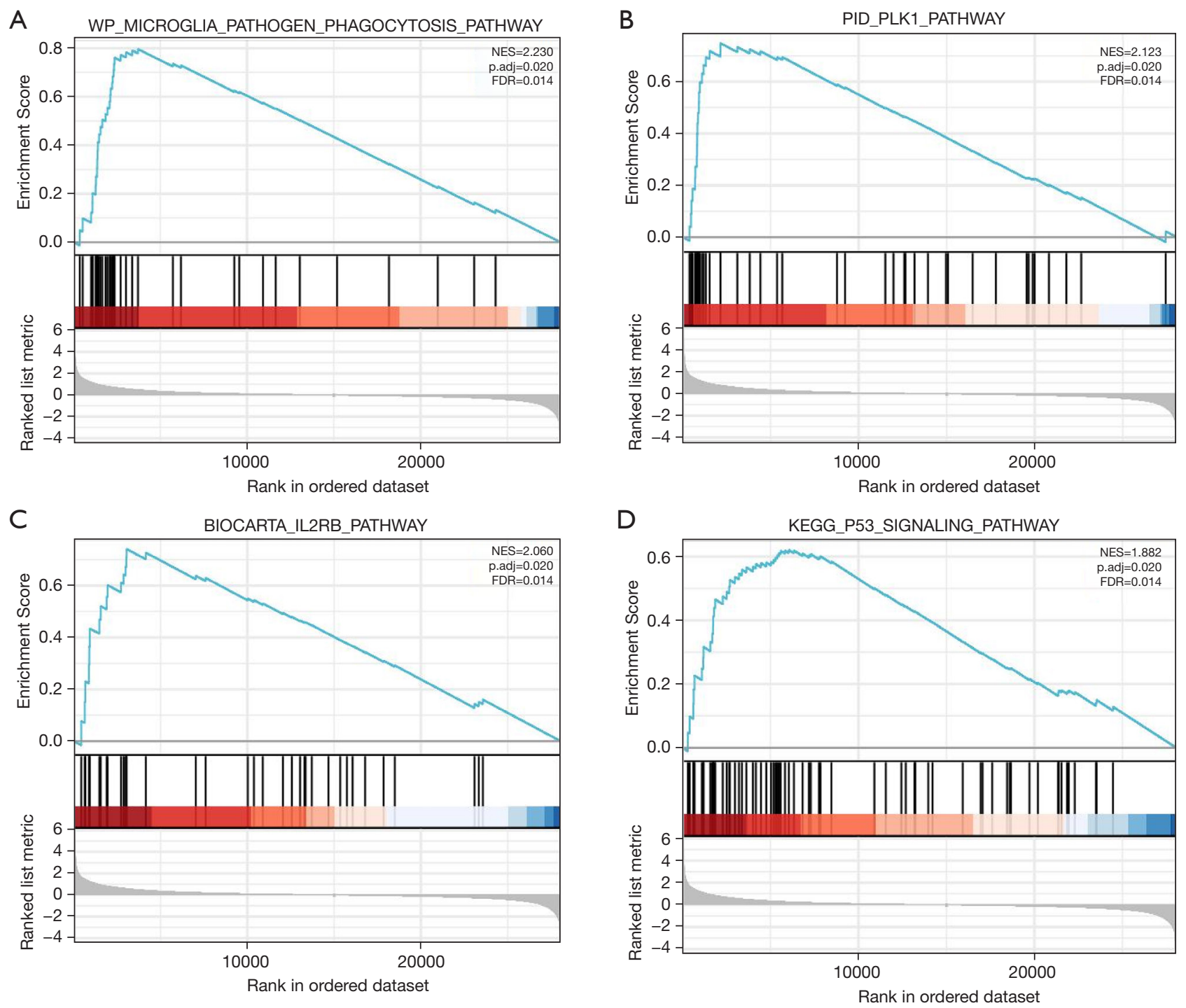

Figure 4 Enrichment plots from GSEA. (A) Microglial pathogen phagocytosis pathway; (B) PLK1 pathway; (C) IL2RB pathway; (D) P53 signal pathway. NES, normalized enrichment score; p-adj, adjusted P value; FDR, false discovery rate; GSEA, gene set enrichment analysis.

Immune checkpoints and single-gene co-expression heat maps suggested that $C M T M 3$ has a significant correlation with CD274, PDCD1, CTLA4, and LAG3 (Figure 6B). Then, we used the TIMER tool to study the association between CMTM3 and the level of tumor immune infiltration (Figure 6C). There were very high correlations shown for CMTM3 and macrophages $(\mathrm{r}=0.536, \mathrm{P}=1.31 \mathrm{e}-36)$, dendritic cell $(\mathrm{r}=0.546, \mathrm{P}=2.85 \mathrm{e}-38)$, and $\mathrm{CD}^{+} \mathrm{T}$ Cell $(\mathrm{r}=0.517$, $\mathrm{P}=6.17 \mathrm{e}-34)$. The above results indicated that the expression of CMTM3 has a high correlation with the level of immune infiltration in WHO grade II and III glioma.

\section{Discussion}

Prognostic markers can effectively evaluate and predict the clinical outcome of patients, and have been widely used in a variety of tumors, such as glioma (13), breast cancer (14), lung cancer (15), renal clear cell carcinoma (16), and others. It can reduce clinical under-treatment or over-treatment. Previous studies have found that CMTM3 is associated with the progression of multiple diseases, and CMTM3 has been associated with male laryngeal squamous cell carcinoma (17). Our study found that the expression of CMTM3 was 
A

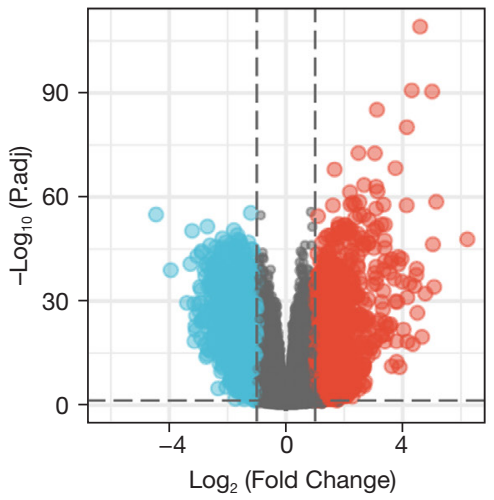

C

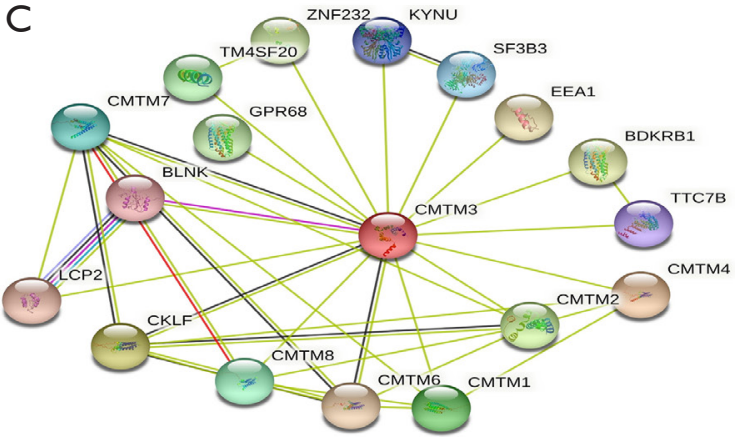

B

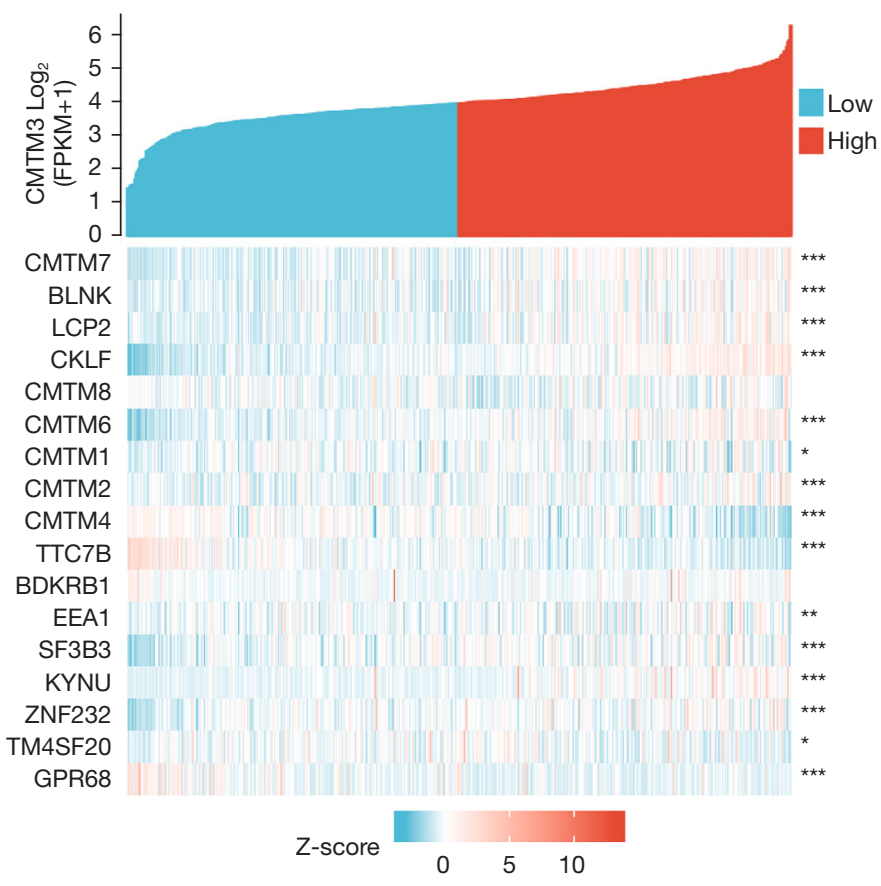

Figure 5 Gene association analysis of CMTM3 in LGG. (A) Volcano map of differentially expressed genes in high and low expression of CMTM3 in LGG; (B) Heat map of CMTM3 and correlated genes; *, $\mathrm{P}<0.05$; **, $\mathrm{P}<0.01$; ${ }^{* *}, \mathrm{P}<0.001$; (C) protein interaction between CMTM3 and related genes. LGG, low grade glioma; CMTM3, CKLF-like MARVEL transmembrane domain-containing 3.

Table 2 Differential gene expression of CMTM3 high expression and low expression groups in LGG

\begin{tabular}{lcc}
\hline Gene name & log $_{2}$ FoldChange & Padj \\
\hline HOXC13 & 5.116668651 & $1.87908 \mathrm{E}-35$ \\
POSTN & 4.959385817 & $3.67362 \mathrm{E}-89$ \\
HOXB8 & 4.705844078 & $1.02491 \mathrm{E}-39$ \\
HOXB3 & 4.657518999 & $9.548 \mathrm{E}-114$ \\
HOXC11 & 4.652442525 & $5.00443 \mathrm{E}-31$ \\
C6orf15 & 4.644090357 & $3.09759 \mathrm{E}-20$ \\
HOXC9 & 4.597175779 & $1.12082 \mathrm{E}-41$ \\
HOXD13 & 4.513324636 & $1.45978 \mathrm{E}-36$ \\
PAX3 & 4.463324487 & $2.21132 \mathrm{E}-40$ \\
HOXB4 & 4.418414293 & $1.07868 \mathrm{E}-97$ \\
HOXC10 & 4.403236267 & $5.69058 \mathrm{E}-42$ \\
OTP & 4.201895569 & $3.88208 \mathrm{E}-53$ \\
GALNT5 & 4.139128918 & $1.12872 \mathrm{E}-80$ \\
HOXD11 & 4.065515911 & $4.95492 \mathrm{E}-23$ \\
HOXD10 & 4.006498818 & $3.4495 \mathrm{E}-44$ \\
\hline
\end{tabular}

Table 2 (continued)
Table 2 (continued)

\begin{tabular}{lcc}
\hline Gene name & log $_{2}$ FoldChange & Padj \\
\hline IBSP & 3.798796221 & $6.08353 \mathrm{E}-45$ \\
VGLL2 & 3.789232212 & $7.14407 \mathrm{E}-40$ \\
HOXC8 & 3.782712612 & $1.64001 \mathrm{E}-30$ \\
PRAC2 & 3.728242979 & $1.68051 \mathrm{E}-26$ \\
HOXA6 & 3.713775102 & $2.59632 \mathrm{E}-18$ \\
HOXC6 & 3.660842717 & $2.36823 \mathrm{E}-44$ \\
HOXC12 & 3.606658037 & $7.77296 \mathrm{E}-12$ \\
TRPM8 & 3.5314238 & $8.05279 \mathrm{E}-68$ \\
ADGRG7 & 3.499388016 & $6.47096 \mathrm{E}-22$ \\
SHOX2 & 3.453481893 & $6.14932 \mathrm{E}-44$ \\
HOXA2 & 3.431057882 & $3.54907 \mathrm{E}-22$ \\
SAA1 & 3.396432996 & $3.61988 \mathrm{E}-33$ \\
HOXB5 & 3.343423128 & $1.31991 \mathrm{E}-40$ \\
IDO1 & 3.324235699 & $3.3799 \mathrm{E}-39$ \\
LBX1 & 3.28583822 & $6.06232 \mathrm{E}-23$ \\
CHI3L1 & 3.265815458 & $1.57582 \mathrm{E}-44$ \\
\hline Table $2(c m i n$ & \\
\hline
\end{tabular}

Table 2 (continued) 
Table 2 (continued)

\begin{tabular}{|c|c|c|}
\hline Gene name & $\log _{2}$ FoldChange & Padj \\
\hline C7orf57 & 3.228683412 & $8.52261 \mathrm{E}-60$ \\
\hline IGF2BP3 & 3.226558417 & $1.57959 E-56$ \\
\hline LTF & 3.217130525 & $2.03887 E-41$ \\
\hline FAM183A & 3.178076455 & $3.10142 \mathrm{E}-41$ \\
\hline CA3 & 3.177462045 & 1.92861E-90 \\
\hline HOXB13 & 3.164652144 & $1.10892 \mathrm{E}-25$ \\
\hline PITX2 & 3.160819249 & 1.73702E-39 \\
\hline HOXA4 & 3.149048616 & 1.14299E-21 \\
\hline SIX6 & 3.140442232 & $8.26446 \mathrm{E}-19$ \\
\hline$A P C D D 1 L$ & 3.095275653 & $1.60446 \mathrm{E}-37$ \\
\hline MEOX2 & 3.07922268 & $2.60697 \mathrm{E}-40$ \\
\hline AGR3 & 3.077692162 & 8.9839E-20 \\
\hline IGF2BP2 & 3.053665798 & 3.91429E-77 \\
\hline C1orf158 & 3.045508525 & 4.60283E-41 \\
\hline DMRTA2 & 3.031489903 & $5.49337 E-52$ \\
\hline HMGA2 & 3.026579035 & 2.01571E-38 \\
\hline PLEKHS1 & 3.018037693 & $2.56356 \mathrm{E}-45$ \\
\hline$N K X 2-5$ & 3.01293066 & 4.70279E-36 \\
\hline EN1 & 3.003903271 & 1.68623E-33 \\
\hline$A B C C 3$ & 2.976055222 & $9.08704 \mathrm{E}-55$ \\
\hline MMP9 & 2.960933955 & $2.33799 E-58$ \\
\hline CHRNA9 & 2.918733041 & 4.14357E-50 \\
\hline HOXA5 & 2.917582715 & 1.77877E-21 \\
\hline WT1 & 2.911836388 & 2.00744E-28 \\
\hline PLA2G2A & 2.893234074 & $2.55948 \mathrm{E}-17$ \\
\hline MAP1LC3C & 2.84670752 & 4.05694E-54 \\
\hline CIBAR2 & 2.840278629 & $3.01785 \mathrm{E}-26$ \\
\hline FOXA2 & 2.839959242 & $7.58558 \mathrm{E}-19$ \\
\hline HOXАЗ & 2.787675112 & $7.43244 \mathrm{E}-16$ \\
\hline HOXD12 & 2.749005069 & $3.78518 \mathrm{E}-14$ \\
\hline$S R Y$ & 2.731640714 & $6.8841 \mathrm{E}-13$ \\
\hline SCNN1B & 2.715860729 & $1.06931 \mathrm{E}-42$ \\
\hline$A C 013470.2$ & 2.67828019 & $6.41459 \mathrm{E}-20$ \\
\hline SPAG17 & 2.66851463 & $5.94338 \mathrm{E}-49$ \\
\hline$S A A 2-S A A 4$ & 2.668349321 & $6.54478 \mathrm{E}-07$ \\
\hline FOXB1 & 2.648331968 & $3.17655 \mathrm{E}-19$ \\
\hline HOXB2 & 2.646456634 & $1.9897 \mathrm{E}-47$ \\
\hline CXCL10 & 2.646342738 & $5.25735 E-45$ \\
\hline IGFBP2 & 2.645651302 & 4.46531E-62 \\
\hline
\end{tabular}

Table 2 (continued)
Table 2 (continued)

\begin{tabular}{|c|c|c|}
\hline Gene name & $\log _{2}$ FoldChange & Padj \\
\hline MAB21L2 & 2.623039832 & 1.44933E-28 \\
\hline HOXA9 & 2.609276712 & $5.44017 \mathrm{E}-18$ \\
\hline IL13RA2 & 2.585659092 & 4.56917E-39 \\
\hline TCTEX1D1 & 2.576035052 & 6.87974E-38 \\
\hline HOXA7 & 2.573732015 & $6.88065 \mathrm{E}-15$ \\
\hline$P D P N$ & 2.572054828 & 1.39163E-57 \\
\hline$F M O D$ & 2.561917386 & $2.9601 \mathrm{E}-48$ \\
\hline PRDM13 & 2.556914175 & 7.32592E-19 \\
\hline WDR38 & 2.550857828 & 1.67817E-38 \\
\hline$D P E P 1$ & 2.549374003 & 3.24019E-43 \\
\hline NNMT & 2.499335554 & 8.63978E-52 \\
\hline GPR139 & 2.493501111 & $6.81191 \mathrm{E}-17$ \\
\hline GPR1 & 2.472611247 & $9.26844 \mathrm{E}-25$ \\
\hline$H P$ & 2.427484569 & 8.13575E-32 \\
\hline H2BC9 & 2.403319338 & 4.74107E-27 \\
\hline ESM1 & 2.401817551 & 5.19493E-30 \\
\hline SPOCD1 & 2.391585749 & 2.70163E-41 \\
\hline$A N X A 1$ & 2.346010961 & $3.83487 \mathrm{E}-57$ \\
\hline$S A A 2$ & 2.3343732 & 1.56859E-27 \\
\hline FAM81B & 2.323524087 & $6.87369 \mathrm{E}-28$ \\
\hline UNCX & 2.300017197 & 3.48162E-06 \\
\hline COL3A1 & 2.296449403 & $6.27604 \mathrm{E}-40$ \\
\hline CFAP45 & 2.296424396 & $5.68643 E-46$ \\
\hline ADAM12 & 2.29075902 & $1.77101 \mathrm{E}-63$ \\
\hline MMP7 & 2.2882843 & $2.34957 \mathrm{E}-25$ \\
\hline TRIM48 & 2.286978985 & 2.76111E-07 \\
\hline FGF3 & 2.284886809 & 1.25874E-05 \\
\hline CXCL9 & 2.278111608 & $2.49079 \mathrm{E}-37$ \\
\hline OTX2 & 2.271472109 & 5.01914E-08 \\
\hline OSR2 & 2.269501718 & $2.13497 \mathrm{E}-25$ \\
\hline IGLL5 & 2.264050095 & $3.70426 \mathrm{E}-15$ \\
\hline PLAU & 2.26009335 & 1.83766E-70 \\
\hline HOXA1 & 2.256048669 & 3.4964E-32 \\
\hline DNAH11 & 2.248773744 & $1.98231 \mathrm{E}-49$ \\
\hline DSG2 & 2.243214101 & 3.75169E-23 \\
\hline PRAME & 2.225962548 & $1.33066 \mathrm{E}-16$ \\
\hline METTL7B & 2.22189128 & 7.62525E-41 \\
\hline CER1 & 2.210057904 & 5.57377E-24 \\
\hline MYBPH & 2.209114505 & $2.11018 \mathrm{E}-51$ \\
\hline
\end{tabular}

Table 2 (continued) 
Table 2 (continued)

\begin{tabular}{|c|c|c|}
\hline Gene name & $\log _{2}$ FoldChange & Padj \\
\hline HOXA10 & 2.203835926 & $4.62318 \mathrm{E}-14$ \\
\hline EMP3 & 2.200164788 & $1.50766 \mathrm{E}-45$ \\
\hline C1orf189 & 2.1971432 & $3.71842 \mathrm{E}-36$ \\
\hline TNFSF14 & 2.176678592 & $1.10875 E-48$ \\
\hline GPX8 & 2.164552937 & $8.62901 E-51$ \\
\hline C11orf88 & 2.155984953 & 8.57172E-32 \\
\hline TMPRSS7 & 2.153415552 & 3.09339E-26 \\
\hline RASEF & 2.152854493 & $1.80182 \mathrm{E}-42$ \\
\hline TIMP1 & 2.143857143 & $1.63342 \mathrm{E}-40$ \\
\hline$H L A-D Q A 2$ & 2.141690362 & $2.74272 \mathrm{E}-26$ \\
\hline HOXB9 & 2.114900842 & $2.14804 \mathrm{E}-13$ \\
\hline CHRNA1 & 2.112533921 & 1.56397E-26 \\
\hline CLEC12A & 2.108393213 & $7.23658 \mathrm{E}-35$ \\
\hline LIF & 2.107516234 & 1.9219E-39 \\
\hline HOXB7 & 2.106584277 & 4.71654E-38 \\
\hline GDF15 & 2.090663327 & $7.08461 \mathrm{E}-35$ \\
\hline TEKT1 & 2.088363812 & $1.42056 \mathrm{E}-31$ \\
\hline ARL14EPL & 2.083088605 & $2.10933 \mathrm{E}-14$ \\
\hline TFAP2B & 2.080092571 & $2.35245 \mathrm{E}-10$ \\
\hline DRC1 & 2.064038168 & 1.5917E-22 \\
\hline COL1A1 & 2.050151005 & $1.96802 \mathrm{E}-33$ \\
\hline FCGBP & 2.031570526 & 1.05493E-41 \\
\hline$A B C B 11$ & 2.023535703 & $1.46968 \mathrm{E}-19$ \\
\hline EDDMЗA & 2.021155161 & 3.87184E-09 \\
\hline ADGRE1 & 2.011136139 & 6.4663E-35 \\
\hline SIM1 & 2.007166223 & $1.36406 \mathrm{E}-08$ \\
\hline APOL4 & 2.003837857 & 8.41203E-40 \\
\hline HOXD9 & 2.003654741 & 4.88096E-14 \\
\hline EYA4 & 2.000149979 & 7.62686E-27 \\
\hline KCNT1 & -2.000425656 & $9.49434 \mathrm{E}-45$ \\
\hline SYT13 & -2.004447708 & 2.04222E-36 \\
\hline MTUS2 & -2.007046581 & $2.9601 \mathrm{E}-48$ \\
\hline$N E F H$ & -2.008667688 & $3.48069 \mathrm{E}-43$ \\
\hline PHF24 & -2.009771634 & 8.32623E-41 \\
\hline TMEM130 & -2.010732944 & 4.90611E-39 \\
\hline KRT3 & -2.011084541 & $1.81158 \mathrm{E}-16$ \\
\hline GPR83 & -2.011221274 & $2.77435 E-36$ \\
\hline KCNB2 & -2.016021649 & $9.25369 \mathrm{E}-33$ \\
\hline KRT31 & -2.016394462 & 2.88513E-24 \\
\hline
\end{tabular}

Table 2 (continued)
Table 2 (continued)

\begin{tabular}{|c|c|c|}
\hline Gene name & $\log _{2}$ FoldChange & Padj \\
\hline GRIN1 & -2.017448395 & 3.04826E-37 \\
\hline GAD2 & -2.017560605 & $9.71647 \mathrm{E}-32$ \\
\hline SERTM1 & -2.029232159 & $1.91892 \mathrm{E}-29$ \\
\hline PTPRR & -2.044425941 & $6.25345 \mathrm{E}-44$ \\
\hline $\mathrm{CRH}$ & -2.046969921 & $1.40043 E-30$ \\
\hline SOHLH1 & -2.052409159 & $1.74515 \mathrm{E}-29$ \\
\hline ATP2B3 & -2.056609799 & 1.76487E-43 \\
\hline TRHDE & -2.058854329 & 1.44864E-34 \\
\hline GSG1L2 & -2.061166978 & 1.47503E-17 \\
\hline C3orf80 & -2.075082068 & $4.52202 \mathrm{E}-42$ \\
\hline HS6ST3 & -2.080328765 & 5.87947E-37 \\
\hline SYNPR & -2.082987743 & 3.71978E-34 \\
\hline$S M P X$ & -2.083532956 & 1.1917E-25 \\
\hline GJB6 & -2.083864735 & $2.89147 \mathrm{E}-23$ \\
\hline $\mathrm{TPH} 2$ & -2.08951245 & 3.38212E-34 \\
\hline EIF4E1B & -2.092218775 & $3.35481 \mathrm{E}-36$ \\
\hline SNAP25 & -2.101842462 & $9.16283 \mathrm{E}-47$ \\
\hline$G D A$ & -2.10403187 & $6.12622 \mathrm{E}-33$ \\
\hline CHRNA2 & -2.104618542 & $8.22836 \mathrm{E}-30$ \\
\hline SLC17A7 & -2.107615563 & $3.92674 \mathrm{E}-29$ \\
\hline FRMPD4 & -2.110734929 & $1.95618 \mathrm{E}-41$ \\
\hline CRYM & -2.111883079 & 7.37337E-33 \\
\hline HTR1A & -2.117518812 & $1.24888 \mathrm{E}-33$ \\
\hline GPR52 & -2.119268119 & 4.98229E-23 \\
\hline SLC22A8 & -2.122612999 & 1.82013E-26 \\
\hline CPNE9 & -2.130126324 & 3.57179E-52 \\
\hline CBLN2 & -2.132829171 & 1.34102E-51 \\
\hline UNC13C & -2.135690586 & $2.36984 \mathrm{E}-37$ \\
\hline$R X F P 1$ & -2.142677783 & 1.13744E-41 \\
\hline GABRA4 & -2.146199486 & $1.51401 \mathrm{E}-34$ \\
\hline STAT4 & -2.149687961 & $8.04074 \mathrm{E}-47$ \\
\hline PCDHGAЗ & -2.149961068 & $9.09778 \mathrm{E}-48$ \\
\hline NWD2 & -2.159704642 & $3.07876 \mathrm{E}-28$ \\
\hline$K L K 5$ & -2.169160593 & 4.79592E-24 \\
\hline CAMK1G & -2.173018216 & $1.45869 \mathrm{E}-33$ \\
\hline SH2D5 & -2.176716893 & $1.66794 \mathrm{E}-46$ \\
\hline LHFPL5 & -2.179416319 & 6.58393E-33 \\
\hline CIDEA & -2.192702209 & $8.20581 \mathrm{E}-35$ \\
\hline BHLHA9 & -2.207783811 & $7.76714 \mathrm{E}-24$ \\
\hline
\end{tabular}

Table 2 (continued) 
Table 2 (continued)

\begin{tabular}{|c|c|c|}
\hline Gene name & $\log _{2}$ FoldChange & Padj \\
\hline$R Y R 2$ & -2.208932724 & $6.30081 \mathrm{E}-42$ \\
\hline MAP7D2 & -2.210547901 & $5.36904 \mathrm{E}-39$ \\
\hline MEPE & -2.223714424 & $3.10448 \mathrm{E}-23$ \\
\hline SSTR4 & -2.226967435 & $3.55716 \mathrm{E}-29$ \\
\hline ANKRD30B & -2.232170903 & $3.2962 \mathrm{E}-41$ \\
\hline$N E F L$ & -2.2343183 & $1.73422 \mathrm{E}-30$ \\
\hline SLC6A17 & -2.237086072 & $1.3379 E-45$ \\
\hline VIP & -2.237640471 & $3.97619 \mathrm{E}-49$ \\
\hline PRKCG & -2.248658823 & 1.11483E-40 \\
\hline IQCF3 & -2.250441258 & $1.87159 \mathrm{E}-17$ \\
\hline OPRM1 & -2.25557997 & $6.8728 \mathrm{E}-25$ \\
\hline TMEM155 & -2.255748661 & $6.02301 \mathrm{E}-40$ \\
\hline SLC12A5 & -2.262088143 & $8.56124 \mathrm{E}-54$ \\
\hline SLC22A9 & -2.266565009 & $2.02681 \mathrm{E}-29$ \\
\hline FAM153B & -2.269323003 & $4.54784 \mathrm{E}-38$ \\
\hline KRT222 & -2.283038129 & $4.75164 \mathrm{E}-46$ \\
\hline SYT1 & -2.286460252 & $1.12829 \mathrm{E}-45$ \\
\hline CREG2 & -2.299541073 & $1.23291 \mathrm{E}-39$ \\
\hline NRGN & -2.300300418 & $2.33789 \mathrm{E}-40$ \\
\hline PNPLA5 & -2.30135478 & $2.37149 \mathrm{E}-32$ \\
\hline SULT4A1 & -2.308305659 & $2.09569 \mathrm{E}-43$ \\
\hline LINC02218 & -2.310141943 & $2.97638 \mathrm{E}-16$ \\
\hline TBR1 & -2.31485039 & $4.4055 \mathrm{E}-42$ \\
\hline LYPD8 & -2.321248192 & 7.35857E-34 \\
\hline$K L K 7$ & -2.324326189 & $9.5428 \mathrm{E}-28$ \\
\hline PVALB & -2.328814713 & $1.37338 \mathrm{E}-31$ \\
\hline$R B F O X 1$ & -2.336287076 & $3.21666 \mathrm{E}-50$ \\
\hline HIPK4 & -2.339576763 & $2.88514 \mathrm{E}-41$ \\
\hline CCK & -2.339668985 & $4.05528 \mathrm{E}-41$ \\
\hline GPR22 & -2.341595608 & $1.85966 \mathrm{E}-43$ \\
\hline PNMA6F & -2.350264426 & $1.51401 \mathrm{E}-34$ \\
\hline PROKR2 & -2.362931824 & 4.45719E-33 \\
\hline HNF4A & -2.36367229 & $1.03574 \mathrm{E}-31$ \\
\hline SDR16C5 & -2.37396754 & $2.37806 \mathrm{E}-40$ \\
\hline RGS4 & -2.379681544 & $9.1091 \mathrm{E}-45$ \\
\hline STYK1 & -2.385309519 & $5.7256 \mathrm{E}-42$ \\
\hline C1QL3 & -2.404478753 & 5.47936E-34 \\
\hline C11orf87 & -2.405081729 & $1.50335 \mathrm{E}-42$ \\
\hline$D D N$ & -2.409162361 & 7.2157E-47 \\
\hline
\end{tabular}

Table 2 (continued)
Table 2 (continued)

\begin{tabular}{|c|c|c|}
\hline Gene name & $\log _{2}$ FoldChange & Padj \\
\hline PACSIN1 & -2.412698014 & $1.29868 \mathrm{E}-42$ \\
\hline SSTR3 & -2.41316214 & 2.18925E-42 \\
\hline MPPED1 & -2.436543 & 5.30699E-46 \\
\hline OLFM3 & -2.440603613 & 4.52253E-50 \\
\hline HTR1E & -2.440659107 & 7.42541E-37 \\
\hline MAL2 & -2.451794291 & 7.89009E-45 \\
\hline CALHM1 & -2.472381369 & 1.77177E-44 \\
\hline CACNG3 & -2.474271337 & 7.39057E-40 \\
\hline HCN1 & -2.476812891 & 7.14877E-46 \\
\hline KCNS2 & -2.504945295 & $5.50985 E-43$ \\
\hline SV2B & -2.524182665 & $9.8185 \mathrm{E}-47$ \\
\hline MAS1 & -2.547665168 & 1.98423E-33 \\
\hline KCNH5 & -2.555303851 & 4.3273E-47 \\
\hline HTR5A & -2.559349382 & 4.31794E-47 \\
\hline TNNT2 & -2.563464962 & $2.43606 \mathrm{E}-37$ \\
\hline GABRA1 & -2.581986894 & 7.40714E-45 \\
\hline$N G B$ & -2.591432149 & $2.30754 \mathrm{E}-40$ \\
\hline VSNL1 & -2.596535624 & 4.75164E-46 \\
\hline THEG & -2.596738717 & $6.28538 E-36$ \\
\hline GABRB2 & -2.613968264 & 2.66929E-52 \\
\hline ANKRD34C & -2.630980278 & $7.98845 E-48$ \\
\hline CAMK2A & -2.631334534 & $4.81438 E-48$ \\
\hline FADS6 & -2.647147861 & $5.12482 E-45$ \\
\hline NEFM & -2.671792682 & $3.92308 \mathrm{E}-47$ \\
\hline SLC6A7 & -2.710308802 & 8.56433E-43 \\
\hline GABRA6 & -2.712321306 & $3.60028 E-39$ \\
\hline KCNV1 & -2.71696464 & 3.365E-37 \\
\hline CNGB1 & -2.769480631 & $3.60028 E-39$ \\
\hline KCNS1 & -2.770816079 & $2.0511 \mathrm{E}-41$ \\
\hline SOWAHB & -2.824115288 & $1.26022 E-58$ \\
\hline ROS1 & -2.876421103 & 8.17676E-42 \\
\hline NEUROD6 & -2.922410812 & $3.14136 \mathrm{E}-43$ \\
\hline GPR26 & -2.949633127 & $7.65748 E-44$ \\
\hline OR14I1 & -2.972889534 & $9.75109 E-33$ \\
\hline NPBWR2 & -3.042387614 & $2.16054 \mathrm{E}-28$ \\
\hline MCHR2 & -3.108660041 & $8.14598 \mathrm{E}-40$ \\
\hline HTR3B & -3.172728665 & $2.33878 \mathrm{E}-48$ \\
\hline TESPA1 & -3.352010134 & $3.06579 E-56$ \\
\hline CTXN3 & -3.55912291 & 1.86724E-50 \\
\hline CARTPT & -4.422650231 & 8.85939E-55 \\
\hline
\end{tabular}

CMTM3, CKLF-like MARVEL transmembrane domain-containing 3; LGG, low grade glioma. 
Table 3 GO and KEGG analysis of differential gene expression in CMTM3 high expression and low expression groups

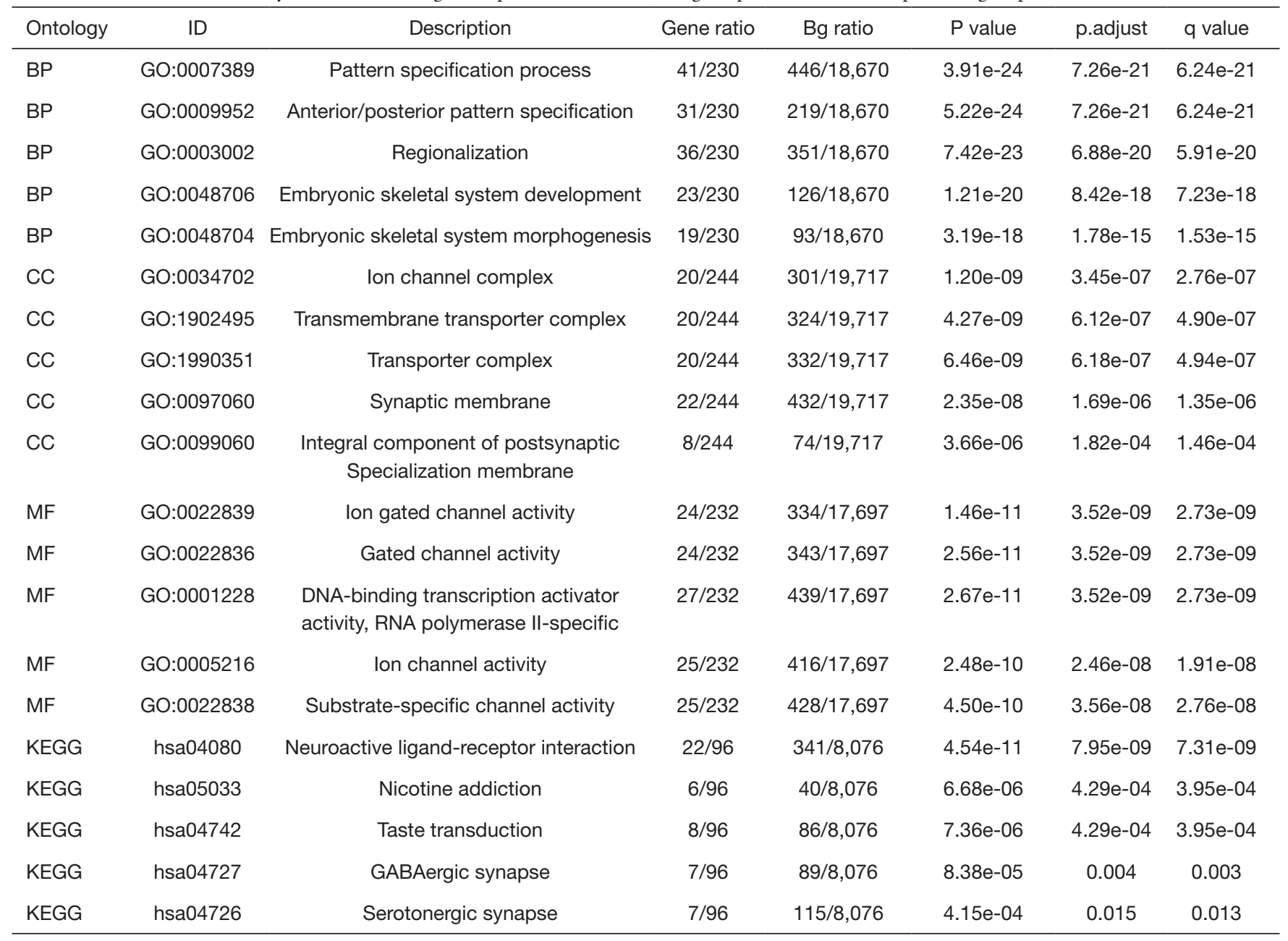

GO, Gene Ontology; KEGG, Kyoto Encyclopedia of Genes Genomes; CMTM3, CKLF-like MARVEL transmembrane domain-containing 3.

significantly increased in glioma, and patients with high CMTM3 expression had a lower OS time. Therefore, it is reasonable to believe that the high expression of CMTM3 will promote the progression of glioma. Subsequently, the relationship between the expression of CMTM3 and the clinical characteristics of glioma was analyzed. The results showed that the expression of CMTM3 in WHO grade III glioma was significantly higher than that in WHO grade II. The IDH wild-type glioma had higher expression of CMTM3 than IDH mutant-type. The expression of CMTM3 in patients with $1 \mathrm{p} / 19 \mathrm{q}$ non-codeletion glioma was significantly higher than that of those with $1 \mathrm{p} / 19 \mathrm{q}$ codeletion. Clinically, patients with WHO grade III, IDH wild-type, and $1 \mathrm{p} / 19 \mathrm{q}$ non-codeletion often have a poor prognosis. Our study showed that the high expression of CMTM3 was closely related to the poor prognosis of glioma. The survival curve showed that the OS of patients with high expression of CMTM3 glioma was significantly lower than that of those with low expression of CMTM3. Therefore, the prognosis of glioma patients can be predicted alternatively by detecting the expression of CMTM3.

Time-dependent ROC can be applied to the prediction of multiple biomarkers. It can predict the survival rate of patients. In this study, the time-dependent ROC curve was drawn to predict the 1- and 3-year survival outcomes of patients, and the AUC was 0.821 and 0.750 . This shows that $C M T M 3$ has high predictive value and can predict the survival of patients with glioma. We used GSEA to evaluate the molecular mechanisms of prognostic genes and determine the related pathways. We also used GSEA to find 4 related pathways in LGG together with high expression of CMTM3. The enrichment results showed that 
A

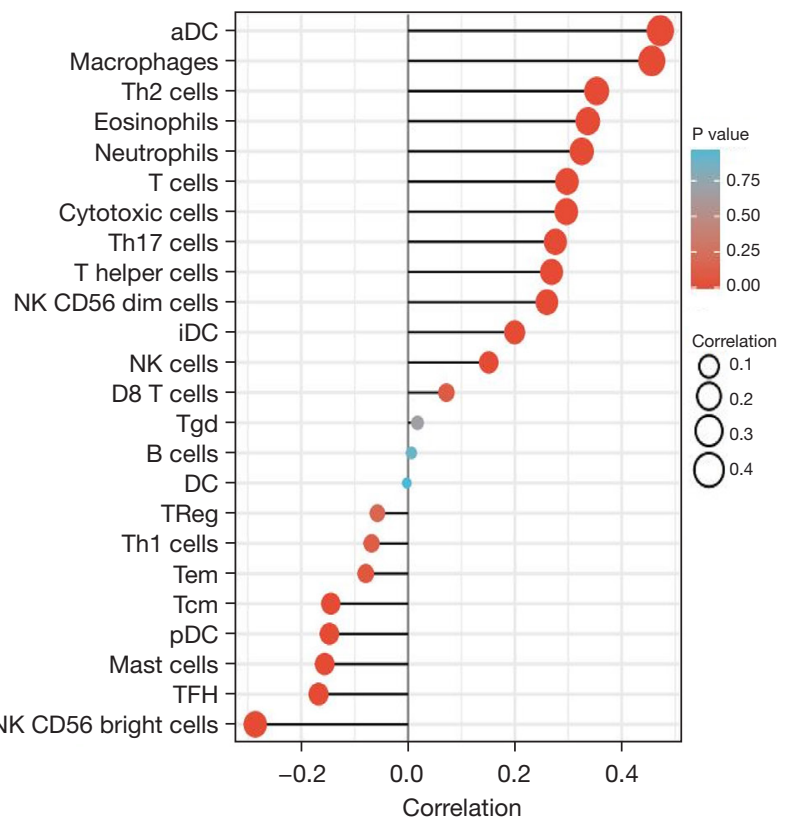

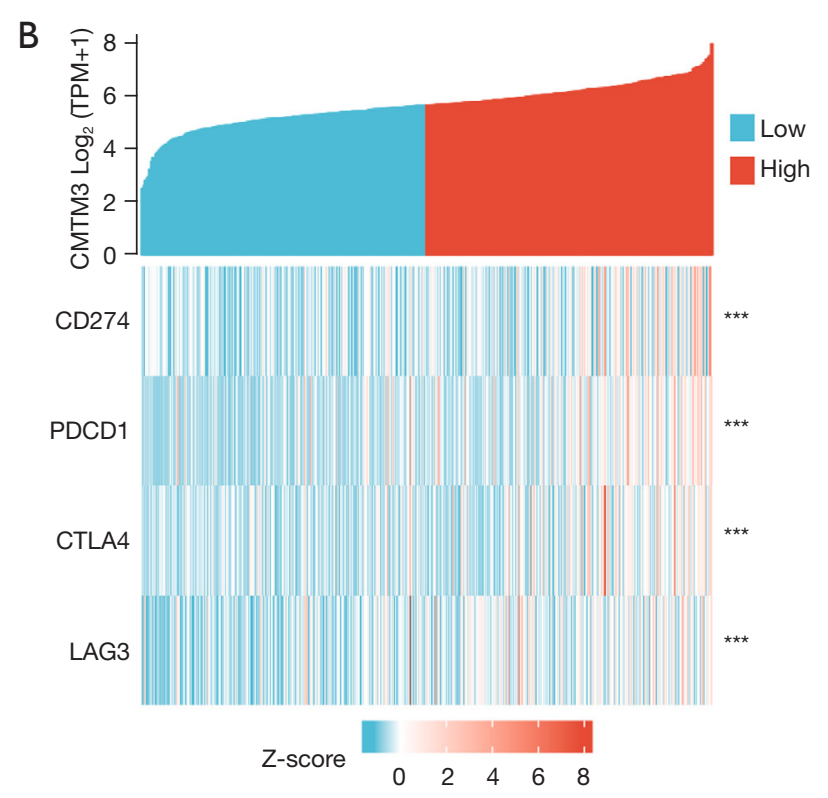

C
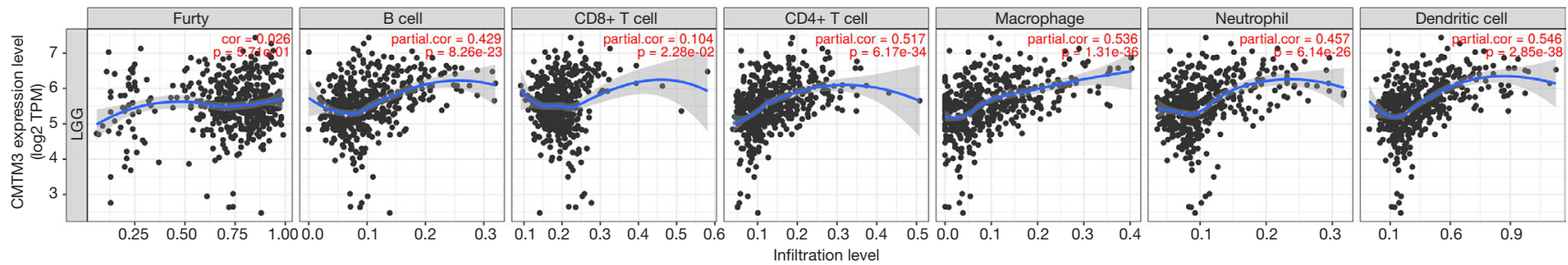

Figure 6 Correlation analysis between CMTM3 and immune cells. (A) ssGSEA immune infiltration algorithm calculated the correlation between CMTM3 and 24 immune cells; (B) immune checkpoints and single-gene co-expression heat maps; ${ }^{* *}, \mathrm{P}<0.001$; (C) correlations between CMTM3 expression and immune infiltration levels in LGG by TIMER database. CMTM3, CKLF-like MARVEL transmembrane domain-containing 3; ssGSEA, single-sample gene set enrichment analysis; LGG, low grade glioma.

CMTM3 was significantly enriched in 4 gene sets, and may affect the progression of glioma through these pathways. These 4 pathways have been shown to be related to many diseases, such as liver fibrosis (18), glioblastoma (19), and so on. Cancer immunotherapy is an important method of treating cancer, in which the patients' own immune system is used to fight tumors (20), thus it has been widely used in the treatment of a variety of tumors (21). Cancer immunotherapy urgently needs biomarkers to accurately predict clinical response, and it has been applied to glioma $(22,23)$. Immune infiltration analysis found that CMTM3 is highly correlated with a variety of immune cells, such as B cell, $\mathrm{CD} 8^{+} \mathrm{T}$ cell, $\mathrm{CD} 4^{+} \mathrm{T}$ cell, macrophage, neutrophil, and dendritic cell. Together, our results indicate that CMTM3 may be a new target for immunotherapy response in glioma. This research was based on bioinformatics and provides new ideas and research directions for follow-up research. It was not a prospective study, so there is the possibility of confounding factors. In future, the specific mechanism of CMTM3 on WHO II and WHO III gliomas needs to be further studied with multi-center experimental data verification.

\section{Conclusions}

Through bioinformatics analyses, we found that the expression of CMTM3 in WHO II and WHO III gliomas was significantly higher than that in normal tissues. The CMTM3 gene is a potential prognostic marker for WHO grade II and III gliomas, and is closely related to the immune infiltration of glioma.

\section{Acknowledgments}

Funding: This work was supported by the youth medical doctors 
project of Jiangsu Province, China (No. QNRC2016870) and Project of Suzhou Health Talents, China (No. 2020090).

\section{Footnote}

Reporting Checklist: The authors have completed the REMARK reporting checklist. Available at https://atm. amegroups.com/article/view/10.21037/atm-22-526/rc

Conflicts of Interest: All authors have completed the ICMJE uniform disclosure form (available at https://atm. amegroups.com/article/view/10.21037/atm-22-526/coif). The authors have no conflicts of interest to declare.

Ethical Statement: The authors are accountable for all aspects of the work in ensuring that questions related to the accuracy or integrity of any part of the work are appropriately investigated and resolved. The study was conducted in accordance with the Declaration of Helsinki (as revised in 2013).

Open Access Statement: This is an Open Access article distributed in accordance with the Creative Commons Attribution-NonCommercial-NoDerivs 4.0 International License (CC BY-NC-ND 4.0), which permits the noncommercial replication and distribution of the article with the strict proviso that no changes or edits are made and the original work is properly cited (including links to both the formal publication through the relevant DOI and the license). See: https://creativecommons.org/licenses/by-nc-nd/4.0/.

\section{References}

1. Weller M, van den Bent M, Preusser M, et al. EANO guidelines on the diagnosis and treatment of diffuse gliomas of adulthood. Nat Rev Clin Oncol 2021;18:170-86.

2. Tan AC, Ashley DM, López GY, et al. Management of glioblastoma: State of the art and future directions. CA Cancer J Clin 2020;70:299-312.

3. Lu M, Huang $Y$, Sun $W$, et al. miR-135b-5p promotes gastric cancer progression by targeting CMTM3. Int J Oncol 2018;52:589-98.

4. Li W, Zhang S. CKLF-Like MARVEL Transmembrane Domain-Containing Member 3 (CMTM3) Inhibits the Proliferation and Tumorigenisis in Hepatocellular Carcinoma Cells. Oncol Res 2017;25:285-93.

5. Hu F, Yuan W, Wang X, et al. CMTM3 is reduced in prostate cancer and inhibits migration, invasion and growth of LNCaP cells. Clin Transl Oncol 2015;17:632-9.

6. Li Z, Xie J, Wu J, et al. CMTM3 inhibits human testicular cancer cell growth through inducing cell-cycle arrest and apoptosis. PLoS One 2014;9:e88965.

7. Zhou Z, Ma Z, Li Z, et al. CMTM3 Overexpression Predicts Poor Survival and Promotes Proliferation and Migration in Pancreatic Cancer. J Cancer 2021;12:5797-806.

8. Yang Q, Wang R, Wei B, et al. Candidate Biomarkers and Molecular Mechanism Investigation for Glioblastoma Multiforme Utilizing WGCNA. Biomed Res Int 2018;2018:4246703.

9. Delic S, Thuy A, Schulze M, et al. Systematic investigation of CMTM family genes suggests relevance to glioblastoma pathogenesis and CMTM1 and CMTM3 as priority targets. Genes Chromosomes Cancer 2015;54:433-43.

10. Li T, Fan J, Wang B, et al. TIMER: A Web Server for Comprehensive Analysis of Tumor-Infiltrating Immune Cells. Cancer Res 2017;77:e108-10.

11. Subramanian A, Kuehn H, Gould J, et al. GSEA-P: a desktop application for Gene Set Enrichment Analysis. Bioinformatics 2007;23:3251-3.

12. Tang Z, Kang B, Li C, et al. GEPIA2: an enhanced web server for large-scale expression profiling and interactive analysis. Nucleic Acids Res 2019;47:W556-60.

13. Kristensen BW, Priesterbach-Ackley LP, Petersen JK, et al. Molecular pathology of tumors of the central nervous system. Ann Oncol 2019;30:1265-78.

14. Cocco S, Piezzo M, Calabrese A, et al. Biomarkers in Triple-Negative Breast Cancer: State-of-the-Art and Future Perspectives. Int J Mol Sci 2020;21:4579.

15. Seijo LM, Peled N, Ajona D, et al. Biomarkers in Lung Cancer Screening: Achievements, Promises, and Challenges. J Thorac Oncol 2019;14:343-57.

16. Lin J, Yu M, Xu X, et al. Identification of biomarkers related to CD8+ T cell infiltration with gene co-expression network in clear cell renal cell carcinoma. Aging (Albany NY) 2020;12:3694-712.

17. Xie J, Yuan Y, Liu Z, et al. CMTM3 is frequently reduced in clear cell renal cell carcinoma and exhibits tumor suppressor activities. Clin Transl Oncol 2014;16:402-9.

18. Chen Y, Chen X, Ji YR, et al. PLK1 regulates hepatic stellate cell activation and liver fibrosis through Wnt/ $\beta$-catenin signalling pathway. J Cell Mol Med 2020;24:7405-16.

19. Lan Y, Lou J, Hu J, et al. Downregulation of SNRPG induces cell cycle arrest and sensitizes human glioblastoma cells to temozolomide by targeting Myc through a p53-dependent signaling pathway. Cancer Biol Med 
2020;17:112-31.

20. Gohil SH, Iorgulescu JB, Braun DA, et al. Applying highdimensional single-cell technologies to the analysis of cancer immunotherapy. Nat Rev Clin Oncol 2021;18:244-56.

21. Meric-Bernstam F, Larkin J, Tabernero J, et al. Enhancing anti-tumour efficacy with immunotherapy combinations. Lancet 2021;397:1010-22.

22. Tang B, Guo ZS, Bartlett DL, et al. Synergistic

Cite this article as: Li S, Gao P, Dai X, Ye L, Wang Z, Cheng H. New prognostic biomarker CMTM3 in low grade glioma and its immune infiltration. Ann Transl Med 2022;10(4):206. doi: 10.21037/atm-22-526
Combination of Oncolytic Virotherapy and Immunotherapy for Glioma. Clin Cancer Res 2020;26:2216-30.

23. Chen L, Qin D, Guo X, et al. Putting Proteomics Into Immunotherapy for Glioblastoma. Front Immunol 2021;12:593255.

(English Language Editor: J. Jones) 